\section{More statistical programs for Commodore computers}

\section{MILTON H. HODGE and DAVID J. PITTENGER University of Georgia. Athens, Georgia}

In a previous article, Pittenger and Hodge (1986) presented a comprehensive package of statistical software for Commodore computers. In the present paper, several revisions and additions are described that enhance the overall utility of the package.

\section{Overview}

The programs are written in BASIC to operate on the Commodore family of computers, including the PET/CBM 4032 and 8032, the C-64, and the C-128. The programs are stored on a disk that is read/write compatible with Commodore 4040, 2031, 1541, and 1571 disk drives. Most of the programs also require a printer.

The Appendix summarizes the major features and analytical options of the package. A description of the revisions and additions is given below. An important component is Data Manager, a utility program that creates, edits, and stores on disk the data files used with the majority of the computational programs. Although the amount of memory in these Commodore machines is less than in other popular computers, it is sufficient for the analysis of typical data sets.

We have attempted to maximize computational accuracy by the use of updating routines (Lachenbruch, 1983) and other algorithms that minimize rounding errors.

\section{Revisions and New Procedures}

Correlation/Regression. Two revisions have been made in this program, described in Pittenger and Hodge (1986). First, we divided the routines into separate parts to obtain more efficient use of computer memory. The Correlation program calculates all possible Pearson correlation coefficients $(r)$ and reports with each coefficient the $t$ ratio and degrees of freedom. The program will also print the mean, standard deviation, sum of scores, and sum of squared scores for each variable. The correlation matrix is saved on disk for use with the Regression program. The Regression program calculates bivariate and multiple linear regression equations. The user must identify the dependent variable and any number of independent variables. The printout includes an estimate of the intercept, the beta weights of each independent variable, $R^{2}$, and an analysis of variance of the regression model.

Requests for reprints and copies of the program disk and user manual may be sent to either author, Department of Psychology, University of Georgia, Athens, GA 30602.
Second, we increased computational accuracy by using $z$ scores to calculate the correlation coefficients. Testing the programs with Longley's (1967) data, we found that all correlation and regression coefficients were error free relative to Longley's computations.

Significance Tester. This program now evaluates the significance of a difference between two independent Pearson correlation coefficients, an observed and population proportion, and two observed proportions.

Multiple Comparisons. This program now includes the Fisher LSD procedure. As with the orthogonal comparisons test, the user may specify the probability level $(.05$ to .005) at which the compared means are to be tested.

One- and Two-Sample Comparisons. This is a new program that contains five separate routines: $t$ test of an observed mean against a population mean, $t$ test of two independent means, $t$ test of two related groups, MannWhitney $U$ test, and Wilcoxon sign test. The program calculates and prints the mean for parametric tests and the median for nonparametric tests, the standard deviation of each group, the value of the test statistic, and the degrees of freedom. The Behrens-Fisher $t$ is also reported when $t$ test for independent groups is selected. The correlation between groups is included with the $t$ test of related means. Both the Mann-Whitney and the Wilcoxon tests are corrected for ties within the data, and when sample sizes are sufficiently large, a $z$ score approximation is given.

Contingency Table Analysis. This new option has two analytical routines. One evaluates $2 \times 2$ (fourfold) tables, and the other analyzes contingency tables with $R$ rows and $C$ columns. Both programs have their own data entry and editing routines. The four-fold program prints the Fisher exact test and chi-square, coefficient kappa (Cohen, 1960), coefficient phi, and effective percent agreement and disagreement (Hartman, 1977). The $R \times C$ table analysis reports the three lamda coefficients (Goodman \& Kruskal, 1954; Hartwig, 1973), chi-square and the corrected contingency coefficient (Sachs, 1984), and the coefficient kappa.

Matrix Algebra. The matrix algebra program is another stand-alone program that performs common matrix algebra operations, including matrix and scalar addition, subtraction, and multiplication; matrix transposition and inversion; and calculation of the determinant.

\section{Documentation and Availability}

In a comprehensive manual for the package, a separate section describes the purpose, capabilities and limitations, and step-by-step use of each program. A copy of the disk and manual may be obtained by sending a check or money order for $\$ 15$ (\$20 U.S. money order for foreign requests) to either author (payable to Psi Chi, UGA Chapter). 


\section{REFERENCES}

ButLer, D. L., \& JoNEs, S. K. (1987). A comparison of inexpensive statistical packages for Apple II microcomputers. Behavior Research Methods, Instruments, \& Computers, 19, 99-103.

COHEN, J. (1960). A coefficient of agreement for nominal scales. Educational \& Psychological Measurement, 20, 37-46.

Goodman, L. A., \& KRUSKAL, W. H. (1954). Measures of association for cross classifications. Journal of the American Statistical Association, 49, 732-764.

Hartman, D. P. (1977). Consideration in the choice of interobserver reliability estimates. Joumal of Applied Behavior Analysis, 10, 103-116.

HARTWIG, F. (1973). Statistical significance of the lambda coefficients. Behavior Science, 18, 307-310.

LACHENBRUCH, P. A. (1983, November). Statistical programs for microcomputers. Byte Magazine, pp. 560-570.

LONGLEY, J. W. (1967). An appraisal of least squares programs for the electronic computer from the point of view of the user. Journal of the American Statistical Association, 62, 819-841.

Pittenger, D. J., \& Hodge, M. H. (1986). Statistical programs for Commodore computers. Behavior Research Methods, Instruments, \& Computers, 18, 50-52.

SACHS, L. (1984). Applied statistics: A handbook of techniques (2nd ed.). New York: Springer-Verlag.

\section{APPENDIX}

Primary Features of the Commodore Statistics Package* General Features

Hardware

Commodore 4032, 8032, 64, 128

RAM

Printer

Cost

Menus

Copy Protected

List Protected

Language

Manual

Overall Error Trapping

\section{Statistics}

Descriptive Statistics

Number of Observations

Arithmetic Mean

Harmonic Mean

Geometric Mean

Median

Mode

Variance

Standard Deviation

Standard Error of Mean
32-64K

Yes

$\$ 15$

Yes

No

No

BASIC

Compiled BASIC

Yes

Yes

See Text

Yes

Yes

Yes

Yes

Yes

Yes

Yes

Yes
Range Yes

Quartiles and Quartile Range Yes

Coefficient of Variation Yes

Skewness and Kurtosis Yes

Frequency Distribution Yes

Correlation

Pearson $r \quad$ Yes

Spearman tho No

Accuracy

Multiple Linear Regression Yes

Simple Comparisons

$t$ Test

Independent Groups

Behrens-Fisher Test

Dependent Groups

Mann-Whitney $U$

Wilcoxon

Analysis of Variance

Independent Variables 9

Repeated Measures Yes

Epsilon Correction Yes

Multiple Comparisons

Orthogonal Comparisons Yes

Fisher LSD Yes

Tukey HSD Yes

Contingency Table Analysis

Chi-Squared

Fisher Exact Test

Coefficient Kappa

Lambda Coefficients

Coefficient Phi

Matrix Algebra

Common Operations

Yes

Yes

Yes

Yes

Yes

Extensive

Other Features

Graphics

Scattergram No

Histogram No

Data Input

Disk Files

Yes

Specific to Package Yes

Compatible Across Routines Yes

Error Trapping Yes

Data Editing

Onscreen Yes

Groups Numbered Yes

Transforming Variables

Extensive

*Characteristics based on Butler and Jones (1987).

(Revision accepted for publication May 18, 1988.) 